\title{
Global measurement of the mesospheric sodium layer by the star occultation instrument GOMOS
}

\author{
D. Fussen, ${ }^{1}$ F. Vanhellemont, ${ }^{1}$ C. Bingen, ${ }^{1}$ E. Kyrölä, ${ }^{2}$ J. Tamminen, ${ }^{2}$ V. Sofieva, ${ }^{2}$ \\ S. Hassinen, ${ }^{2}$ A. Seppälä, ${ }^{2}$ P. Verronen, ${ }^{2}$ J.-L. Bertaux, ${ }^{3}$ A. Hauchecorne, ${ }^{3}$ F. Dalaudier, ${ }^{3}$ \\ J.-B. Renard, ${ }^{4}$ R. Fraisse, ${ }^{5}$ O. Fanton d'Andon, ${ }^{6}$ G. Barrot, ${ }^{6}$ A. Mangin, ${ }^{6}$ B. Théodore, ${ }^{6}$ \\ M. Guirlet, ${ }^{6}$ R. Koopman, ${ }^{7}$ P. Snoeij, ${ }^{7}$ and L. Saavedra ${ }^{7}$ \\ Received 27 September 2004; revised 22 November 2004; accepted 3 December 2004; published 31 December 2004.
}

[1] We present the first global measurement of the sodium mesospheric layer obtained from the processing of about 100000 star occultations by the GOMOS instrument onboard the ENVISAT satellite. The retrieval method is developed on the basis of a simple DOAS retrieval applied to averaged transmittances. The vertical inversion of the sodium slant path optical thickness is performed by using a modified Gaussian extinction profile. A global climatology is derived by using monthly bins of 20 degrees in latitude. The high variability of the sodium layer is confirmed on a global scale as well as the presence of an important modulation in the annual cycle. Also, we present some evidence for the existence of a diurnal cycle characterized by an increase of the sodium concentration in daylight. INDEX TERMS: 0340 Atmospheric Composition and Structure: Middle atmosphere-composition and chemistry; 0360 Atmospheric Composition and Structure: Transmission and scattering of radiation; 1640 Global Change: Remote sensing. Citation: Fussen, D., et al. (2004), Global measurement of the mesospheric sodium layer by the star occultation instrument GOMOS, Geophys. Res. Lett., 31, L24110, doi:10.1029/ 2004 GL021618.

\section{Introduction: The GOMOS Experiment}

[2] The ENVISAT satellite was launched by the European Space Agency (ESA) on March 1, 2002 and put into a heliosynchronous circular orbit at an altitude of $800 \mathrm{~km}$. Amongst the experiments devoted to the study of the Earth's atmosphere, the GOMOS (Global Ozone Monitoring by Occultation of Stars) instrument was the first to exploit routinely the principle of stellar occultations [Kyrölä et al., 2004]. Briefly, the GOMOS spectrometers are coupled to a telescope system capable of tracking star settings. The absolute transmittance along the line of sight can be obtained by taking the ratio of the spectrum measured

\footnotetext{
${ }^{1}$ Institut d'Aéronomie Spatiale de Belgique, Brussels, Belgium.

${ }^{2}$ Earth Observation Department, Finnish Meteorological Institute, Helsinki, Finland.

${ }^{3}$ Service d'Aéronomie du CNRS, Verrières-le-Buisson, France.

${ }^{4}$ Laboratoire de Physique et Chimie de l'Environnement, Orléans, France.

${ }^{5}$ Astrium, Toulouse, France.

${ }^{6}$ Analytic and Computational Research, Inc.-Earth Sciences (ACRI-ST), Sophia-Antipolis, France. Italy.

${ }^{7}$ European Space Research Institute, European Space Agency, Frascati,
}

Copyright 2004 by the American Geophysical Union. 0094-8276/04/2004GL021618 through the atmosphere, to the extraterrestrial reference. The instrument is clearly self-calibrating and offers a major advantage over the solar occultation technique in the number of available occultations (20 to 40 per orbit selected in the subset of the 200 brightest stars) although the signal-tonoise ratio is less, requiring an integration time of about 0.5 second $(1.7 \mathrm{~km}$ sampling resolution for an occultation in the orbital plane).

[3] Starting from geolocated transmittances, the production of useful level 2 data requires both spectral and vertical inversions where the relative contributions of different absorbing species and different atmospheric layers are respectively retrieved. The spectral ranges of the GOMOS spectrometers $(250-690 \mathrm{~nm}, 750-776 \mathrm{~nm}, 916-956 \mathrm{~nm})$ span the UV-VIS-IR domain and were chosen to mainly target the $\mathrm{O}_{3}, \mathrm{NO}_{2}, \mathrm{NO}_{3}, \mathrm{H}_{2} \mathrm{O}, \mathrm{O}_{2}$ concentration and aerosol extinction profiles.

[4] Several hundreds of thousands of occultations have been successfully performed so far and the data of the entire year 2003 are presently available, on which this work is based. Apart from the spectral signature of the main atmospheric absorbers, a careful inspection of the measured GOMOS transmittances revealed the presence of an absorption doublet at $589 \mathrm{~nm}$ that could be unambiguously attributed to mesospheric atomic sodium. Although extensive data exist about the mesospheric sodium layer measured from particular sites, very little is known about the geographical distribution [Plane, 2004]. Besides the scientific questions related to the origin and the vertical distribution of the layer, the mesospheric sodium is of major importance in astronomy where it is used to produce virtual stars for adaptive optics. This letter presents the first global mesospheric sodium climatology measured so far.

\section{Retrieval of the Sodium Number Density Profile}

[5] In view of the low signal-to-noise ratio inherent to the stellar occultation technique, the retrieval of weak absorption features is a difficult task, especially when observing faint stars in bright limb conditions. Therefore, keeping in mind the construction of a global climatology, it is useful to average (with proper statistical factors) within time-latitude bins several hundreds to several thousands of transmittances after reduction to a common altitude grid. The retrieval of the sodium slant path optical thickness can be performed by using a simple DOAS technique (see Figure 1). Indeed, at tangent altitudes above $60 \mathrm{~km}$, the GOMOS transmittance is not affected by the structured absorption in the ozone 


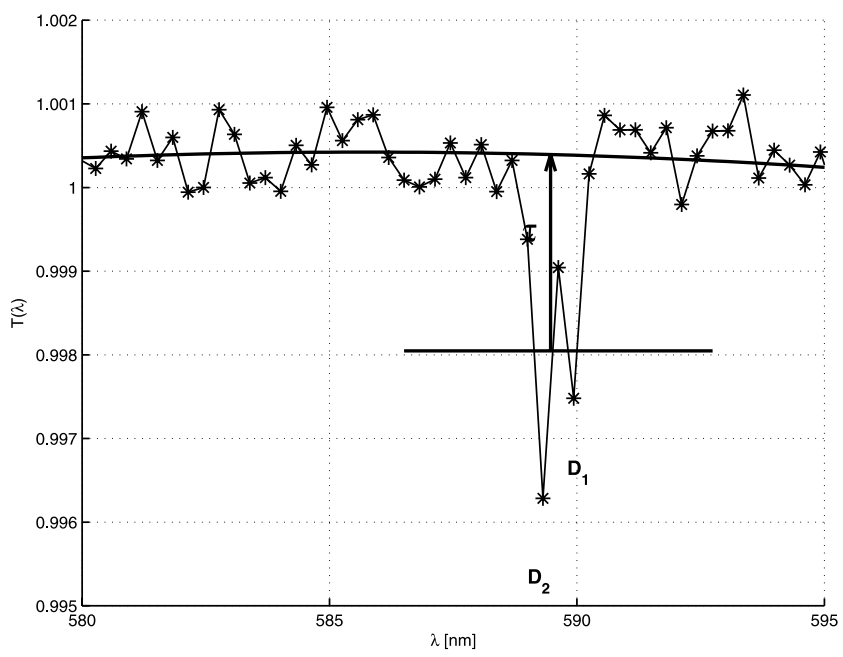

Figure 1. Averaged GOMOS transmittance (from 2023 occultations) between $10^{\circ} \mathrm{N}$ and $30^{\circ} \mathrm{N}$ for Sept. 2003. The tangent altitude is $88 \mathrm{~km}$. The D2-D1 absorption doublet correspond to the fine structure splitting of the $3^{2} \mathrm{~S}-3^{2} \mathrm{P}$ transition and $\tau$ is the $\mathrm{Na}$ mean optical thickness with respect to the baseline (full line).

Chappuis band. Hence, the optical thickness of the sodium D1-D2 atomic absorption lines, can easily be extracted by fitting the baseline transmittance in the window [579-588] $\cup$ [591-599] $\mathrm{nm}$ by a second order polynomial. The procedure was however repeated twice, the first time to allow the detection of possible outliers, the second time to compute the baseline with outliers removed. The quadratic wavelength dependence of the baseline was necessary to take into account the limb contribution in daylight occultations.

[6] As the D1-D2 fine structure splitting is not always well resolved, we decided to evaluate the mean absorption over 4 pixels spanning a wavelength range of about $1.25 \mathrm{~nm}$. The natural cologarithm of the differential transmittance evaluated within this interval is the sodium slant path optical thickness at the considered tangent altitude.

[7] Detailed calculation of the total photoabsorption cross section by sodium atoms have been published by [Fricke and Von Zahn, 1985] who were interested in the sensitivity of the hyperfine structure splitting (convolved by the Doppler distribution) to the temperature. Such a spectral accuracy $(0.002 \mathrm{~nm})$ is beyond the capabilities of the GOMOS spectrometer resolution but the effective integrated cross section is rather insensitive to the temperature. In the case of the GOMOS instrument, the high-resolution cross section has to be convolved with the instrument line shape which has a FWHM of about $0.53 \mathrm{~nm}$ at the considered wavelength. Assuming a mean mesospheric temperature of $200 \mathrm{~K}$, and considering that the D1 cross section is half the D2 value, calculations lead to the effective cross section:

$$
\sigma=2.1210^{-14} \mathrm{~cm}^{2}
$$

[8] It is possible to approximate the $\mathrm{Na}$ concentration profile by a Gaussian shape $n_{G}(z)$ expressed by

$$
n_{G}(z)=n_{0} \exp \left(-\left(\frac{z-z_{0}}{H}\right)^{2}\right)
$$

where $n_{0}, z_{0}$ and $H$ respectively refer to the Na concentration $\left[\mathrm{cm}^{-3}\right]$, the altitude of the maximum $[\mathrm{km}]$ and the distribution width $[\mathrm{km}]$. Multiplying the last equation by the cross section $\sigma$, one gets the extinction profile $\beta(z)$ as

$$
\beta_{G}(z)=\beta_{0} \exp \left(-\left(\frac{z-z_{0}}{H}\right)^{2}\right)
$$

[9] In the mesosphere, refraction is negligible and for a given tangent altitude $h$, stellar rays propagate along a rectilinear coordinate $x$. Using elementary geometry for a spherical Earth of radius $R$, we have

$$
z-z_{0}=\sqrt{\rho^{2}+x^{2}}-(\rho-\delta)
$$

where $\rho=R+h$ and $\delta=h-z_{0}$. If $\delta<0$, the extinction profile possesses three extrema along the optical path and it is necessary to keep the $\rho^{4}$ term in the approximation of equation (4). Neglecting $\delta$ with respect to $\rho$, the slant path optical thickness $\tau$ can be analytically integrated as

$$
\begin{gathered}
\tau \simeq \int_{-\infty}^{\infty} \beta_{G}(z(x)) d x \\
\tau \simeq \beta_{0} 2^{\frac{1}{4}} \sqrt{\pi H\left(R+z_{0}\right)} \exp \left(-\frac{\delta^{2}}{2 H^{2}}\right) D_{\frac{-1}{2}}\left(\frac{\sqrt{2} \delta}{H}\right)
\end{gathered}
$$

where $D_{\frac{-1}{2}}\left(\frac{\sqrt{2} \delta}{H}\right)$ is the parabolic cylinder function [Abramowitz and Stegun, 1972]. Differentiating the last equation, one finds that the altitude of the maximum of the optical thickness is displaced by about the half-width of the absorbing layer as

$$
\delta * \simeq-0.541 H
$$
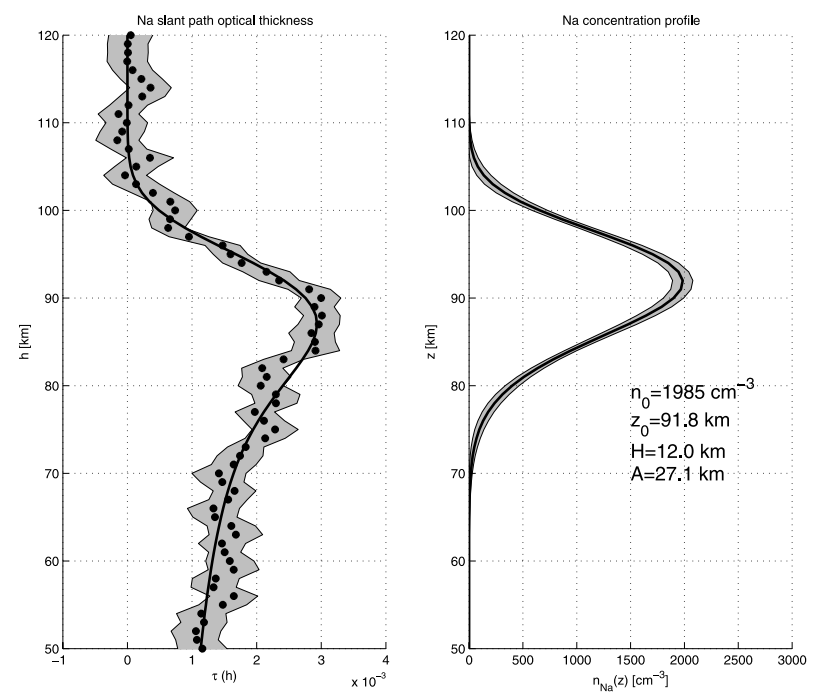

Figure 2. Same geolocation as Figure 1. Left panel: Retrieved Na slant path optical thickness (full circles) versus the tangent altitude with associated errors (gray band). The full line is the best fit obtained from equation (9). Right panel: corresponding $\mathrm{Na}$ concentration profile and optimal parameters. The gray band represents the estimated random error on the transmittance (see Figure 1). 


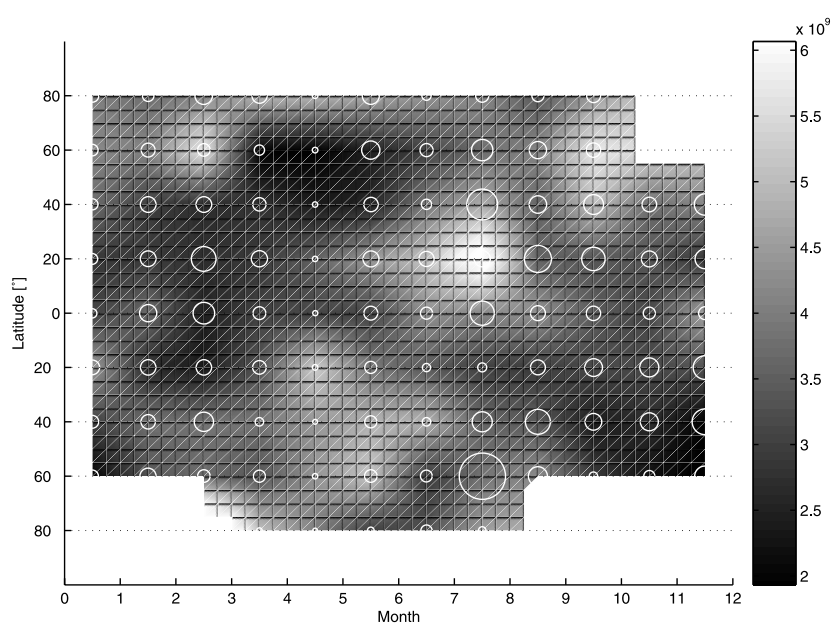

Figure 3. The Na column as measured by GOMOS in 2003. The units on the color bar are in $\mathrm{cm}^{-2}$. The size of the circles represents a linear mapping of the number of aggregated occultations per bin (ranging between 100 and 3000). See color version of this figure in the HTML.

and the corresponding optical thickness value is

$$
\tau(\delta *)=3.044 \beta_{0} \sqrt{H\left(R+z_{0}\right)}
$$

[10] In the left panel of Figure 2, the evolution of the slant path sodium optical thickness versus tangent altitude clearly shows the characteristics of an absorbing layer with an abrupt edge above $95 \mathrm{~km}$ and a slow decrease below the maximum around $88 \mathrm{~km}$.

[11] However, there are theoretical arguments [Plane et al., 1999; Mégie and Blamont, 1977] to introduce some asymmetry in the sodium extinction profile. Hence, we have

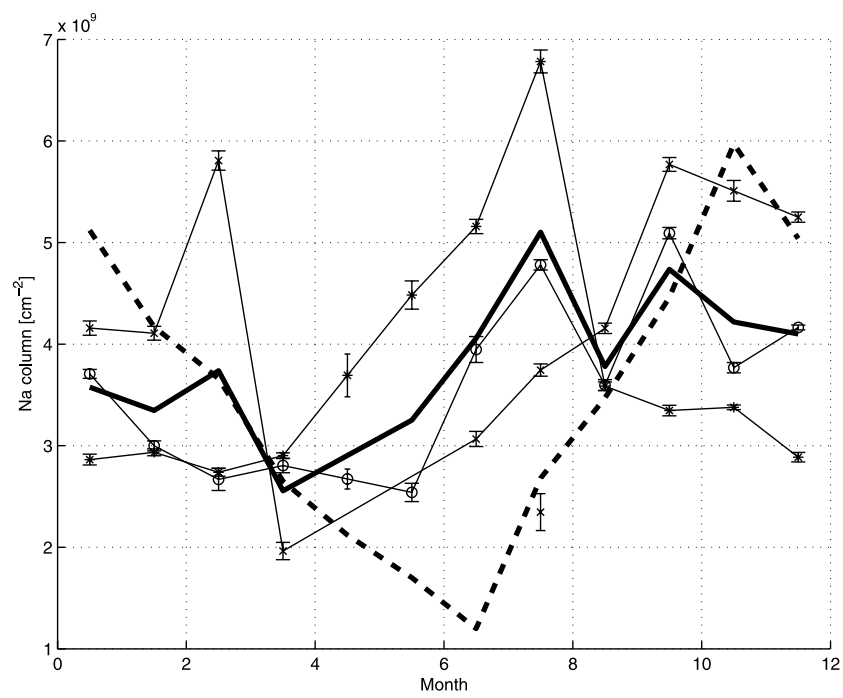

Figure 4. Thin lines: temporal variation of the Na column retrieved from GOMOS data in latitude bands centered at $20^{\circ} \mathrm{N}$ (stars), $40^{\circ} \mathrm{N}$ (circles) and $60^{\circ} \mathrm{N}$ (crosses). The full thick line is the mean to be compared to the Fort Collins data (dashed line). used a modified Gaussian extinction profile (with a supplementary asymmetry factor $A$ )

$$
\beta(z)=\sigma n_{0} \exp \left(-\left(\frac{z-z_{0}}{H}\right)^{2}\left[1+\exp \left(-\left(\frac{z-z_{0}}{A}\right)\right)\right]\right)
$$

for the construction of a fit merit function that quadratically compares the difference (weighted by the experimental errors) between the numerically integrated $\beta(z)$ and the experimentally retrieved values of $\tau(h)$ in the $50-120 \mathrm{~km}$ altitude range. The merit function was minimized by a standard Levenberg-Marquardt algorithm and the profile estimated error was computed from the Jacobian. The error refers to the random uncertainty of the averaged transmittance and not to the unknown natural variability of the considered bin. A typical inversion result is presented in the right panel of Figure 2.

\section{The Sodium Layer in 2003}

[12] It is useful to compute the sodium column density by integrating the concentration profile along the vertical direction (Figure 3). Clearly, an significant variability is observed but the most striking feature is probably the broad minimum of the Na column occurring during April-May in the northern hemisphere and shifted to Oct-November in the southern hemisphere.

[13] In Figure 4, we compare the temporal evolution of the Na column densities in $20^{\circ}$ bands centered at 20,40 and 60 degrees of North latitudes with the climatological results presented by [She et al., 2000]. There is a reasonable agreement concerning the column magnitudes and the general time evolution with a spring decrease followed by a fall-winter increase. The minimum in the Fort Collins climatology is however attained in June-July and it is very difficult to decide whether this is a systematic difference or a particular pattern of the year 2003 (the natural variability of the Fort Collins measurements is about $40 \%$ at the maximum of $\mathrm{Na}$ concentration profile). The latitude dependence of this temporal evolution may be a very sensitive parameter. The time-altitude $\mathrm{Na}$ concentration contour plot

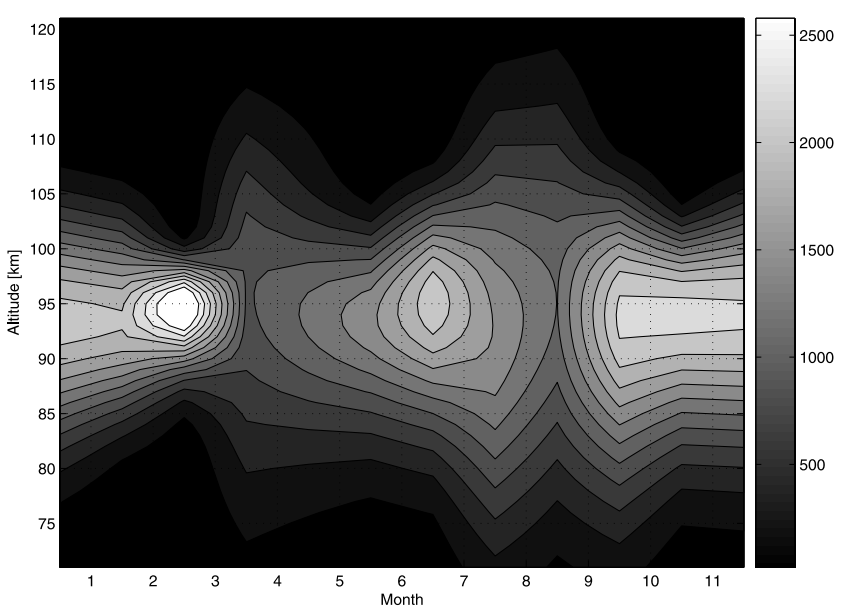

Figure 5. The time-altitude $\mathrm{Na}$ concentration (in $\mathrm{cm}^{-3}$ ) retrieved from GOMOS data in the $40^{\circ} \mathrm{N}$ latitude band. See color version of this figure in the HTML. 
Table 1. Mean Parameters (Year 2003) of the Na Concentration Profile Defined by Equation (9)

\begin{tabular}{cccc}
\hline$n_{0}$ & $z_{0}$ & $H$ & $A$ \\
\hline $1970 \pm 510$ & $92.8 \pm 1.9$ & $14.5 \pm 4.7$ & $36.3 \pm 77.5$ \\
$\mathrm{~cm}^{-3}$ & $\mathrm{~km}$ & $\mathrm{~km}$ & $\mathrm{~km}$ \\
\hline
\end{tabular}

at $40^{\circ}$ is shown in Figure 5 and exhibits a time evolution compatible with sophisticated models [Plane et al., 1999]. The annually averaged maximal Na concentration (at $92 \mathrm{~km}$ ) measured by GOMOS is $1970 \pm 510 \mathrm{~cm}^{-3}$, which is about $30 \%$ lower than the Fort Collins values $\left(2980 \pm 1070 \mathrm{~cm}^{-3}\right)$. The highest $\mathrm{Na}$ peak concentration measured by GOMOS reached $4080 \mathrm{~cm}^{-3}$ in the Antarctic regions. In Table 1, we report the mean sodium concentration profile averaged for the entire year 2003.

[14] Although a large number of dark and bright limb occultations occur at around $23 \mathrm{~h}$ and $9 \mathrm{~h}$ respectively, GOMOS has measured events at any local time (LT) of the tangent point. This allows to sample the diurnal evolution of the sodium layer. In Figure 6, we report the sodium slant path optical thickness profiles measured in 4 temporal windows having a 6 hour width. A noon/dawn/twilight increase of the sodium as large as $50 \%$ is observed between $70 \mathrm{~km}$ and $90 \mathrm{~km}$ but the noon value below $70 \mathrm{~km}$ seems unrealistically small probably due to insufficient statistics. A related dependence on the solar zenith angle (SZA) is shown in Figure 7. According to the most recent models, the $\mathrm{Na}$ increase in daylight could be attributed to the photolysis of the reservoir species $\mathrm{NaHCO}_{3}$ and this is perhaps the first time that this effect is experimentally observed, preventing the necessity of introducing the $\mathrm{NaHCO}_{3}$ dimerization and the uptake of sodium species onto meteoritic smoke particles as was hypothesized by [Plane, 2004].

\section{Conclusions}

[15] The GOMOS instrument turned out to be able to detect very tenuous constituents like the mesospheric sodium. Slant path optical thicknesses as small as 0.001 can be retrieved with a reasonable accuracy by using

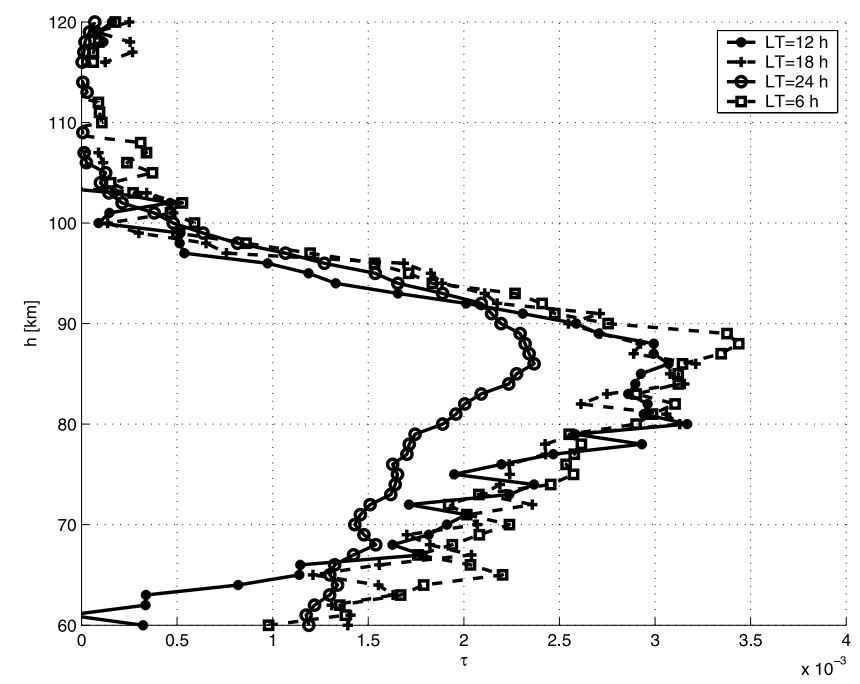

Figure 6. Averaged $\mathrm{Na}$ slant path optical thicknesses for different local times: LT $=12 \mathrm{~h}$ (dots), LT $=18 \mathrm{~h}$ (crosses), $\mathrm{LT}=24 \mathrm{~h}$ (circles) and LT $=6 \mathrm{~h}$ (squares).

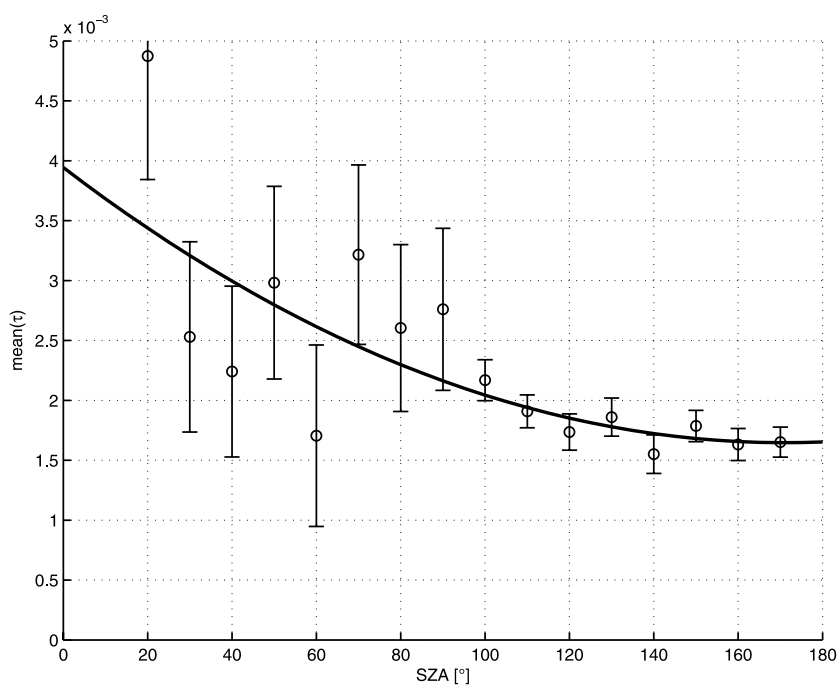

Figure 7. Median Na slant path optical thicknesses as a function of the solar zenith angle SZA at the tangent point. The full line is a simple weighted quadratic fit through the data.

cumulative transmittances. A global coverage with monthly and 20-degree bins may be achieved on the statistics of about 100000 occultations.

[16] The results will be consolidated in a near future by the addition of 10 months of data for the year 2002 and data measured after January 2004, which are not yet available. In particular, the unusual activity of some meteoritic showers in 2002 may have left a signature in the sodium data field.

[17] The $\mathrm{Na} 2003$ data presented here have already confirmed the strong mesospheric variability. They suggest the presence of an important annual cycle in both hemispheres and also a systematic increase of the sodium abundance in daylight conditions.

[18] Acknowledgment. This work has been partly supported by the PRODEX 7 project "SADE" and by the research project MO/35/009 under the authority of the Belgian Federal Science Policy Office.

\section{References}

Abramowitz, M., and I. A. Stegun (1972), Handbook of Mathematical Functions, Dover, Mineola, New York.

Fricke, K. H., and U. Von Zahn (1985), Mesopause temperatures derived from probing the hyperfine structure of the $\mathrm{D}_{2}$ resonance line, J. Atmos. Terr. Phys., 47, 449-512.

Kyrölä, E., et al. (2004), GOMOS on Envisat: An overview, Adv. Space Res., 33, 1020-1028.

Mégie, G., and J. E. Blamont (1977), Laser sounding of atmospheric sodium: Interpretation in terms of global atmospheric parameters, Planet. Space Sci., 25, 1093-1109.

Plane, J. M. C. (2004), A time-resolved model of the mesospheric Na layer: Constraints on the meteor input function, Atmos. Chem. Phys., 4, 627638.

Plane, J. M. C., C. S. Gardner, J. Yu, C. Y. She, R. Garcia, and H. C. Pumphrey (1999), Mesospheric Na layer at $40^{\circ} \mathrm{N}$ : Modeling and observations, J. Geophys. Res., 104, 3773-3788.

She, C. Y., S. Chen, Z. Hu, J. Sherman, J. D. Vance, V. Vasoli, M. A. White, Y. Yu, and D. A. Krueger (2000), Eight-year climatology of nocturnal temperature and sodium density in the mesopause region (80 to $105 \mathrm{~km}$ ) over Fort Collins, $\mathrm{CO}\left(41^{\circ} \mathrm{N}, 105^{\circ} \mathrm{W}\right)$, Geophys. Res. Lett., 27, 32893292 .

D. Fussen, F. Vanhellemont, and C. Bingen, Institut d'Aéronomie Spatiale de Belgique, 3, avenue Circulaire, B-1180, Bruxelles, Belgium. (didier.fussen@oma.be) 
E. Kyrölä, J. Tamminen, V. Sofieva, S. Hassinen, A. Seppälä, and P. Verronen, Earth Observation Department, Finnish Meteorological Institute, P.O. Box 503, FIN-001010 Helsinki, Finland.

J.-L. Bertaux, A. Hauchecorne, and F. Dalaudier, Service d'Aéronomie du CNRS, BP 3, F-91371 Verrières-le-Buisson, France.

J.-B. Renard, Laboratoire de Physique et Chimie de l'Environnement, A, avenue de la Recherche Scientifique, F-45071, Orléans cedex 2, France.
R. Fraisse, Astrium, 31, avenue des Cosmonautes, F-31402 Toulouse cedex 4, France.

O. Fanton d'Andon, G. Barrot, A. Mangin, B. Théodore, and M. Guirlet, ACRI-ST, 260, route du Pin Montard, BP 234, F-06904 Sophia-Antipolis, France.

R. Koopman, P. Snoeij, and L. Saavedra, ESRIN, ESA, Via Galileo Galilei, I-00044 Frascati, Italy. 